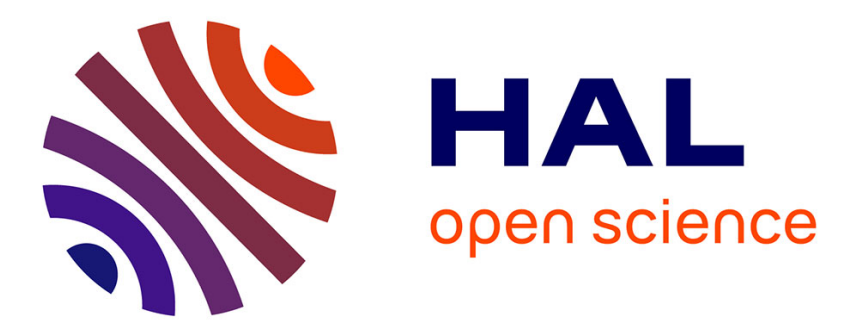

\title{
Caractérisation du fonctionnement d'un ouvrage de gestion à la source du ruissellement de voirie: approche par modélisation hydrologique et par cartographie de la contamination du sol
}

Kelsey Flanagan, Damien Tedoldi, Philippe Branchu, Marie-Christine

Gromaire

\section{To cite this version:}

Kelsey Flanagan, Damien Tedoldi, Philippe Branchu, Marie-Christine Gromaire. Caractérisation $\mathrm{du}$ fonctionnement d'un ouvrage de gestion à la source du ruissellement de voirie: approche par modélisation hydrologique et par cartographie de la contamination du sol. La Houille Blanche Revue internationale de l'eau, 2017, 3, pp.5-13. 10.1051/lhb/2017016 . hal-01570255

\section{HAL Id: hal-01570255 \\ https: / hal-enpc.archives-ouvertes.fr/hal-01570255}

Submitted on 28 Jul 2017

HAL is a multi-disciplinary open access archive for the deposit and dissemination of scientific research documents, whether they are published or not. The documents may come from teaching and research institutions in France or abroad, or from public or private research centers.
L'archive ouverte pluridisciplinaire HAL, est destinée au dépôt et à la diffusion de documents scientifiques de niveau recherche, publiés ou non, émanant des établissements d'enseignement et de recherche français ou étrangers, des laboratoires publics ou privés. 


\title{
Caractérisation du fonctionnement d'un ouvrage de gestion à la source du ruissellement de voirie : approche par modélisation hydrologique et par cartographie de la contamination du sol
}

\author{
Kelsey FLANAGAN², Damien TEDOLDI ${ }^{1}$, Philippe BRANCHU ${ }^{3}$, Marie-Christine GROMAIRE ${ }^{1}$
}

\author{
${ }^{1}$ Laboratoire Eau, Environnement, Systèmes Urbains (LEESU) - ENPC, 6-8 Avenue Blaise Pascal, Cité Descartes, Champs-sur-Marne. 77455 \\ Marne-la-Vallée Cedex 2 -e-mail: kelsey.flanagan@enpc.fr, damien.tedoldi@enpc.fr \\ ${ }^{2}$ SEPIA, 53 rue de Turbigo, 75003 Paris \\ ${ }^{3}$ CEREMA Trappes, 12 Rue Léon Teisserenc de Bort, 78190 Trappes
}

\begin{abstract}
RÉSUMÉ. - La mise en œuvre d'une gestion décentralisée des eaux pluviales implique généralement le recours à des systèmes d'infiltration, dont le fonctionnement n'est pas toujours correctement appréhendé. Une mauvaise évaluation de l'hydrologie peut s'avérer préjudiciable pour la gestion quantitative et qualitative du ruissellement, notamment dans des dispositifs conçus pour une maitrise «à la source » des flux de polluants. Face aux difficultés associées à la mesure directe des flux d'eau et de polluants sur des ouvrages en place, on discute les apports de deux méthodes indirectes, appliquées à un site réel constitué d'une bande enherbée suivi d'un fossé filtrant: l'utilisation d'un modèle hydrologique et l'étude de la pollution du sol.

Le modèle hydrologique présente des incertitudes dues à ses hypothèses simplificatrices, ainsi qu'à la variabilité des mesures de conductivité hydraulique. Il fournit toutefois un ordre de grandeur de la sollicitation relative des différentes parties de l'ouvrage. La cartographie des contaminants, qui montre que la majeure partie de la pollution s'accumule à proximité de la chaussée, renseigne indirectement sur la répartition " intégrée dans le temps » des flux d'infiltration et des processus de sédimentation/filtration sur le site d'étude. L'utilisation d'une résolution spatiale suffisamment fine permet de mettre en évidence des hétérogénéités dans l'infiltration et les écoulements superficiels.

Malgré des limites propres à chaque approche, les deux méthodes mènent à la conclusion qu'une grande partie des volumes ruisselés - et, de facto, du flux polluant - est gérée par infiltration dans la bande enherbée, la partie de l'ouvrage à proximité immédiate de la zone d'arrivée de l'eau dans l'ouvrage. Ainsi, le fossé filtrant ne joue pas son rôle épuratoire de manière optimale, dans la mesure où les contaminants seraient retenus en bordure de chaussée dans le sol de surface.
\end{abstract}

Mots-clés : Contamination, distribution spatiale, gestion à la source, ruissellement, SWMM

\section{Characterization of the behavior of a sustainable urban drainage system treating road runoff through hydrologic modeling and soil contamination cartography}

\begin{abstract}
Decentralized stormwater management generally involves the use of infiltration systems, the comportment of which is not always correctly understood. Incorrect assumptions about the hydrologic behavior of such systems can undermine their roles in both the quantitative and qualitative management of runoff, especially in systems designed to treat pollution at the source. Given the technical difficulty of directly measuring water fluxes in already existing systems, this paper discusses the utility of two indirect methods, applied to a real site made up of a vegetative filter strip followed by a biofiltration swale: the use of a hydrologic model and the characterization of soil pollution.

The hydrologic model involves uncertainties due to its simplifying hypotheses and the variability of soil hydraulic conductivity measurements. Still, it can provide a rough estimation of the relative frequency of infiltration in different parts of the system. The cartography of contaminants, which shows that the majority of pollution is accumulated close to the roadside, indirectly provides time-integrated information as to the repartition of infiltration fluxes and the processes of sedimentation and filtration at the study site. The high spatial resolution of soil contamination data also provides evidence of heterogeneous surface runoff and infiltration flows.

Despite the limits of each approach, both methods lead to the conclusion that the majority of runoff volumes - and as a consequence, the majority of pollutant loads - is infiltrated in the part of the system nearest to the system inlet: the vegetative filter strip. Therefore, the biofiltration swale does not fulfill its initially intended role of depolluting runoff water, as most pollutants do not reach it but rather are retained in the surface soil close to the roadside.
\end{abstract}

Key-words: Contamination, spatial distribution, sustainable urban drainage systems, runoff, SWMM

\section{INTRODUCTION}

Depuis quelques décennies, le domaine de l'hydrologie urbaine évolue vers de nouveaux paradigmes. Les objectifs de la gestion des eaux pluviales, initialement limités à la collecte et l'évacuation afin d'éviter les inondations, se sont étendus à la protection de la qualité des milieux récepteurs, au maintien d'un bilan hydrologique similaire à celui pré-urbanisation, et à la valorisation des eaux pluviales en ville pour contribuer à la qualité de vie [Fletcher et al., 2014]. L'approche classique 
du « tout tuyau » se trouve ainsi progressivement suppléé par des " techniques alternatives " favorisant la gestion à l'amont des eaux pluviales. En France, la mise en place de ces techniques répond généralement à des critères réglementaires imposant une limitation des débits admissibles à l'entrée des réseaux collectifs. A cet effet, l'infiltration (totale ou partielle) de l'eau dans le sol est une technique souvent privilégiée - du moins lorsque le contexte et les enjeux hydrogéologiques le permettent [Petrucci, 2012].

$\mathrm{Au}$ regard du potentiel de pollution des eaux de ruissellement, il devient aujourd'hui assez courant d'intégrer une dimension d'épuration à ces systèmes, en visant une maîtrise « à la source » du flux polluant. Ces ouvrages, dits de « biorétention » ou « biofiltration », sont conçus dans l'idée d'optimiser les processus naturels de dépollution ayant lieu dans l'écosystème du sol. Ils consistent en un espace de stockage au fond duquel se trouve un substrat filtrant planté [Davis et al., 2009 ; LeFevre et al., 2014], et sont souvent intégrés dans une chaîne de dispositifs [Bastien et al., 2010].

La compréhension et l'anticipation correcte du fonctionnement hydrologique de ces ouvrages pour les événements pluvieux courants, responsables de la majorité du flux polluant à l'échelle annuelle, apparaissent comme un prérequis indispensable à (i) une conception optimisée pour l'abattement des flux de contaminants, (ii) une évaluation pertinente de leurs performances épuratoires, ainsi que (iii) une identification claire des besoins de maintenance. Or cette compréhension n'est pas aisément acquise, étant donné que la mesure directe des flux d'eau et de polluants est difficilement possible à moins que l'ouvrage n'ait été étanché et équipé d'un drain dès sa conception. La mise en place de capteurs hydrologiques demeure possible, mais difficile, et l'interprétation des données issues de ces capteurs nécessite le recours à un modèle du site [Ramier, 2005]. De ce fait, il peut s'avérer utile de s'appuyer sur des méthodes indirectes pour aborder la question du fonctionnement hydrologique et d'épuration de tels ouvrages. À cet effet, on considère ici deux approches différentes mais complémentaires, appliquées à un ouvrage réel, à la géométrie simple, constitué d'une bande enherbée et d'un fossé filtrant, conçu pour une gestion quantitative et qualitative du ruissellement de voirie.

Premièrement, un modèle hydrologique est mis en œuvre, dans lequel la bande enherbée et le talus du fossé sont représentés de façon très simplifiée avec un nombre limité de paramètres physiques. Par ailleurs, une cartographie détaillée des niveaux de contamination en cuivre, plomb et zinc est réalisée. Ces trois métaux ont été sélectionnés comme traceurs de la pollution chronique d'origine routière, en raison de leur caractère persistant dans les sols et de leurs propriétés physico-chimiques contrastées ; ce sont par ailleurs les contaminants les plus fréquemment suivis dans les systèmes d'infiltration des eaux de ruissellement urbaines [Tedoldi et al., 2016]. Ces deux méthodes, très différentes, peuvent chacune contribuer à une meilleure compréhension du fonctionnement hydrologique de l'ouvrage avec certaines limitations. Ce travail explore les apports et les limites des deux approches, montrant ainsi l'intérêt de coupler un travail de modélisation avec un travail expérimental pour mieux caractériser les flux dans un ouvrage.

\section{MATERIELS ET MÉTHODES}

Site d'étude. Le dispositif étudié se situe en bordure de la Route Départementale 212 à Compans (77), et a été mis en place en 2012. Il est constitué d'une bande enherbée, suivie d'un fossé filtrant (un filtre planté linéaire) et récupère les eaux de ruissellement de deux voies de circulation avec un trafic moyen de 11000 véhicules/jour (Figure 1). Le site est équipé d'une glissière de sécurité entre la route et la bande enherbée, présente sur toute la longueur de l'ouvrage.

\section{Tableau 1: Caractéristiques des bandes enherbées des deux segments étudiés}

\begin{tabular}{|l|c|c|}
\cline { 2 - 3 } \multicolumn{1}{c|}{} & Segment 1 & Segment 2 \\
\hline Longueur $\mathbf{( m )}$ & 48 & 48 \\
\hline Type de sol & Sablo-limoneux & Limon argileux \\
\hline Drainage & Nappe drainante & Non drainé \\
\hline $\begin{array}{l}\text { Conductivité } \\
\text { hydraulique }\left(\mathbf{K}_{\mathbf{s}}, \mathbf{m m} / \mathbf{h}\right)\end{array}$ & $24(10-28)$ & $14(1-21)$ \\
\hline
\end{tabular}

L'ouvrage comprend deux segments avec des bandes enherbées aux caractéristiques différentes (Tableau 1). La conductivité hydraulique $\left(\mathrm{K}_{\mathrm{s}}\right)$, dont le Tableau 1 fournit la valeur moyenne par segment ainsi que les valeurs extrêmes, a fait l'objet de trois estimations pour le premier segment et quatre estimations pour le second [Kanso, 2015]. Une nappe drainante, formée de deux tuyaux percés espacés de $0,5 \mathrm{~m}$ entre deux couches de géotextile, est également présente sous le fossé filtrant du segment 1. La pluviométrie annuelle moyenne est de $647 \mathrm{~mm}$ (donnée du Conseil Départemental du 77). Le site a été conçu de façon à ce que l'eau de la voirie arrive sur la bande enherbée par ruissellement direct, qu'elle y transite avant d'atteindre le talus, puis le fond du fossé où elle est censée s'infiltrer, dans un milieu favorisant divers processus de dépollution. Toutefois, de nombreuses observations de terrain suggèrent que la majeure partie du ruissellement s'infiltre dans la bande enherbée et n'atteint pas le massif filtrant du fossé pour les événements pluvieux courants.

Modélisation hydrologique du site. L'objectif principal de ce travail est de mieux comprendre la répartition des flux d'eau entre les différentes sous-parties de l'ouvrage sur le long terme. Pour ce faire, il est nécessaire de représenter la production et le transfert du ruissellement de surface sur la route, la bande enherbée et le talus pour une chronique longue. A cet effet, le système est modélisé à l'aide du logiciel Storm Water Management Model (SWMM 5.1), développé par 1'United States Environmental Protection Agency [Rossman, 2015]. Comme préconisé par Gironás et al. [2009], le système est représenté comme une série de trois sous-bassins versants rectangulaires correspondant respectivement à la route, à la bande enherbée, et au talus du fossé (Figure 1). On considère que le massif filtrant au fond $\mathrm{du}$ fossé, non représenté par le modèle, est la sortie du talus.

Pour chaque bassin versant, ce modèle représente, de façon simplifiée, le ruissellement superficiel, l'infiltration, et l'évapotranspiration, à partir de conditions aux limites atmosphériques et d'un nombre limité de paramètres physiques. Chaque sous-bassin est représenté comme un réservoir non-linéaire avec le bilan hydrologique suivant :

$$
\frac{d h}{d t}=p+r_{e}-e-i-r_{s}
$$

où $h$ est la hauteur d'eau sur le sous bassin versant [L], $p$ est l'intensité des précipitations [L.T $\left.\mathrm{T}^{-1}\right], r_{e}$ est l'arrivée d'eau par

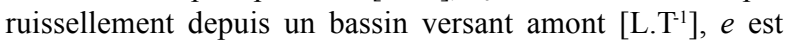

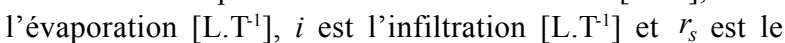
ruissellement sortant du sous-bassin versant $\left[\mathrm{L}^{\mathrm{T}} \mathrm{T}^{1}\right]$. 


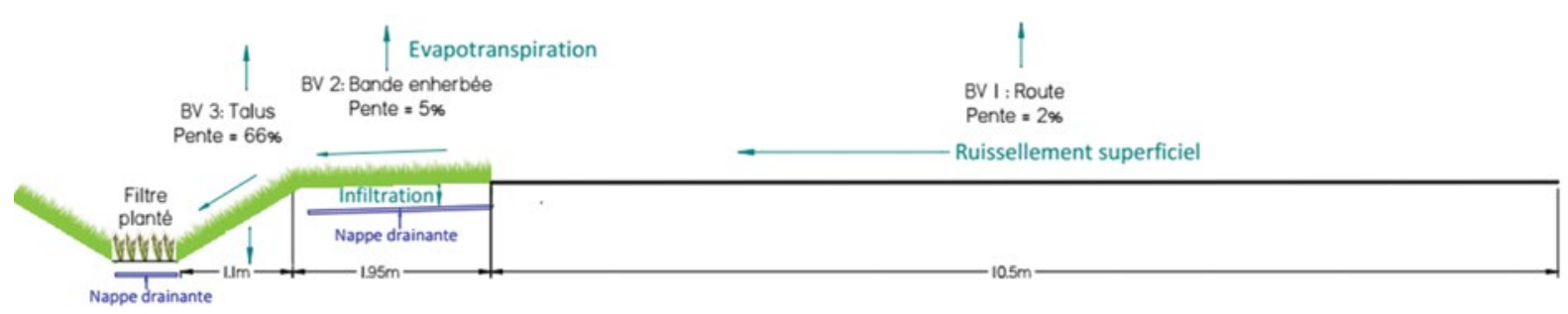

Figure 1 : Géométrie du système étudié. Délimitation des sous-bassins versants représentés par le modèle. Les nappes drainantes ne sont présentes que sur le premier segment de l'ouvrage.

Les précipitations $p$ sont issues d'une chronique réelle de pluie mesurée sur quatre ans en Île-de-France à un pas de temps de 5 minutes. Le terme $e$, estimé à partir de données d'évapotranspiration potentielle couvrant la même période, affecte uniquement l'eau stockée en surface. L'infiltration est calculée par l'équation de Green et Ampt, en faisant l'hypothèse d'une colonne de sol semi-infinie et homogène. Un front de saturation se propage depuis la surface au cours d'un événement pluvieux. A l'arrêt des précipitations, la teneur en eau du sol tend vers sa valeur initiale à une vitesse qui dépend de la conductivité hydraulique du sol. Le ruissellement sortant du bassin est nul tant que la hauteur d'eau $h$ est inférieure à une hauteur de stockage en dépression $h_{s}$, distincte pour chaque sous-bassin versant. Une fois ce stockage dépassé, le terme $r_{s}$ est calculé par l'équation de Manning-Strickler:

$$
r_{s}=\alpha\left(h-h_{s}\right)^{5 / 3}
$$

où $\alpha$ est un paramètre qui dépend de la géométrie du site et de la rugosité de la surface. Le ruissellement est ensuite dirigé vers un sous-bassin versant aval, ou vers un émissaire du système.

Le Tableau 2 présente les valeurs des paramètres communes à toutes les simulations ; $h_{s}$ et la rugosité sont des valeurs typiques des surfaces considérées [Rossman, 2015]. On a supposé que le sol était initialement à sa capacité au champ ; la succion correspondante a été calculée à partir de la courbe de rétention du sol.

Échantillonnage du sol. Les campagnes de terrain ont été entreprises entre Novembre 2015 et Janvier 2016. Afin de caractériser l'étendue latérale et verticale de la contamination sur la bande enherbée, un tronçon de $18 \mathrm{~m}$ de longueur a été échantillonné sur chacun des deux segments. Au cours de la première phase, un échantillon de sol superficiel (1-2 $\mathrm{cm})$ a été prélevé tous les $1,5 \mathrm{~m}$, à des distances de 0,30 , 70,120 , et $180 \mathrm{~cm}$ de la route, à l'aide d'une pelle en acier inoxydable (Figure 2). Les échantillons collectés à $0 \mathrm{~cm}$ correspondent à du dépôt accumulé en bordure de chaussée. Par

Tableau 2 : Paramètres du modèle hydrologique

\begin{tabular}{|c|c|c|c|}
\hline \multirow{2}{*}{ Sous-bassin versant } & \multirow{2}{*}{ Route } & \multicolumn{2}{|c|}{ Surfaces végétalisées } \\
\cline { 3 - 4 } & Segment 1 & Segment 2 \\
\hline $\begin{array}{c}\text { Stockage } \\
\text { en dépression (mm) }\end{array}$ & 1 & 5 & 5 \\
\hline Rugosité & 0,011 & 0,15 & 0,15 \\
\hline $\begin{array}{c}\text { Succion dans la zone } \\
\text { non-saturée (mm) }\end{array}$ & - & 56,3 & 91,1 \\
\hline $\begin{array}{c}\text { Déficit initial de } \\
\text { saturation (\%) }\end{array}$ & - & 17 & 17 \\
\hline
\end{tabular}
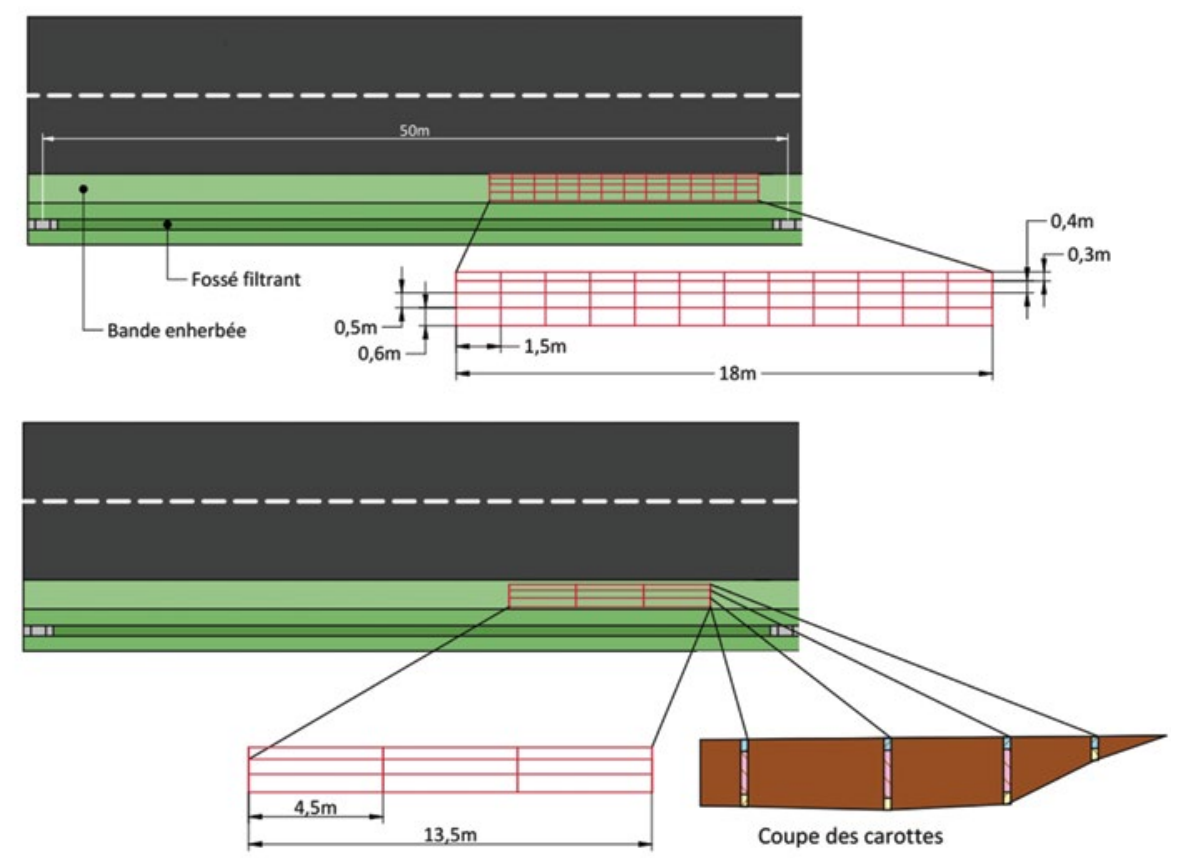

Figure 2 : Schéma du plan d'échantillonnage (a) à la surface et (b) en profondeur 
ailleurs, des prélèvements supplémentaires ont été réalisés sur trois transects d'un segment témoin, non influencé par l'infiltration du ruissellement de voirie en raison de la présence d'une bordure en béton entre la route et l'accotement végétalisé. La seconde phase a consisté en une série d'échantillonnages en profondeur, jusqu'à la nappe drainante (rencontrée à une profondeur variable, $10-18 \mathrm{~cm}$ ) au niveau du segment 1 , et jusqu'à la structure de la route $(10-35 \mathrm{~cm})$ au niveau du segment 2 . Sur les deux tronçons échantillonnés en phase 1 , des carottes ont été prélevées tous les $4,5 \mathrm{~m}$, à des distances de $30,70,120$, et $180 \mathrm{~cm}$ de la route, à l'aide d'une tarière-gouge manuelle. Chaque carotte a été découpée en 2 ou 3 sous-échantillons, de manière à conserver les $5 \mathrm{~cm}$ de surface, les $5 \mathrm{~cm}$ les plus profonds, et le cas échéant la zone intermédiaire (Figure 2). Les outils d'échantillonnage ont été nettoyés et rincés deux fois à l'eau ultra-pure entre deux prélèvements. Les échantillons de sol ont été conservés dans des flacons individuels neufs en polyéthylène haute densité.

Analyses en laboratoire. Conformément à ce que préconise la norme ISO 11464 (2006), les échantillons ont été rapportés au laboratoire, séchés à l'étuve $\left(40^{\circ} \mathrm{C}\right)$ pendant 7 jours, avant d'être broyés manuellement et tamisés à $2 \mathrm{~mm}$ avec une maille en nylon. Les teneurs en cuivre, plomb, et zinc, ont été déterminées par spectrométrie de fluorescence X (Thermo Scientific, Analyseur Niton ${ }^{\mathrm{TM}}$ XL3t) sur des sous-échantillons homogénéisés. Les limites de quantification dépendent du signal reçu par l'analyseur, mais demeurent toujours inférieures à 25,10 , et $30 \mathrm{mg} / \mathrm{kg}$ pour le cuivre, le plomb, et le zinc, respectivement. Quatre mesures ont été réalisées sur différents sous-échantillons, afin de vérifier la répétabilité des valeurs obtenues ; lorsque le coefficient de variation était supérieur à $15 \%$, deux mesures supplémentaires ont été faites. Dans tout ce qui suit, on n'a conservé que les concentrations moyennes par échantillon.

Interpolation spatiale. Des cartographies des concentrations en cuivre, plomb, et zinc, à la surface de chaque tronçon échantillonné, ont été réalisées par interpolation bilinéaire le long d'une grille de $10 \mathrm{~cm} \times 10 \mathrm{~cm}$ à l'aide du logiciel R. Par ailleurs, en faisant l'hypothèse d'une décroissance linéaire des concentrations avec la profondeur, au sein de chaque échantillon "moyen » (d'épaisseur 5 à $20 \mathrm{~cm}$ ) issu de la phase de carottages, on a affecté à chaque concentration la profondeur moyenne de l'échantillon ; ce faisant, il a été possible de cartographier la contamination en métaux lourds en chaque section transversale de la bande enherbée, via la même méthode d'interpolation que précédemment.

\section{RESULTATS ET DISCUSSION}

Simulations hydrologiques. La Figure 3 présente le bilan hydrologique simulé pour une chronique de 4 ans typique du climat d'Ile-de-France, en affectant à chaque segment sa conductivité hydraulique moyenne. Rappelons que le modèle reproduit uniquement l'évaporation de l'eau à la surface, et ignore l'évapotranspiration de l'eau une fois dans le sol. Ce flux d'infiltration est majoritaire au niveau de la bande enherbée : il représente $84 \%$ et $77 \%$ du volume total arrivant sur la bande enherbée pour les premier et second segments respectivement. Une fraction plus petite de l'eau arrivant sur le talus s'y infiltre (59\% et $52 \%$ respectivement). Au bout $\mathrm{du}$ compte, seule une faible proportion du volume d'eau total atteint le fond du fossé filtrant $(10 \%$ et $15 \%)$. Ce résultat suggère une divergence notable entre le fonctionnement effectif de l'ouvrage et ce que prévoyait sa conception initiale, à savoir un traitement du flux polluant dans le fossé filtrant, à un emplacement qui ne reçoit a priori qu'une faible fraction de ce flux.

Comme mentionné précédemment, les mesures de $\mathrm{K}_{\mathrm{s}}$ sur ce site présentent une variabilité importante, provenant en partie de l'incertitude de mesure, et en partie d'une réelle hétérogénéité de $\mathrm{K}_{\mathrm{s}}$ sur site. Or, la structure du modèle impose que $r_{s}$ soit non nul uniquement quand $p+r_{e}$ dépassent les pertes $i+e$ (sachant que $e$ est généralement petit par rapport à $i$ ), et ce pendant une période suffisante pour que la hauteur de stockage en dépression soit dépassée. La relation entre l'intensité de la pluie et la vitesse d'infiltration dans le sol est donc déterminante pour la quantité de ruissellement simulée en sortie d'un bassin versant. Afin de mieux caractériser l'effet de cette variabilité sur l'incertitude du bilan hydrologique à long terme, la proportion de l'eau infiltrée sur la bande enherbée a été simulée pour la chronique de pluie en faisant varier $\mathrm{K}_{\mathrm{s}}$ entre ses valeurs extrêmes (Figure 4). On observe que ce résultat est plus sensible à une variation de $\mathrm{K}_{\mathrm{s}}$ pour de faibles valeurs que pour de fortes valeurs. Cela s'explique par la fréquence plus élevée de pluies de faible intensité, pouvant s'infiltrer intégralement même pour de faibles valeurs de $\mathrm{K}_{\mathrm{s}}$, par rapport aux pluies de plus forte intensité. Ainsi, le pourcentage du flux infiltré au niveau de la bande enherbée varie entre $70-85 \%$ pour le segment 1 et $33-83 \%$ pour le segment 2 .

Des analyses de sensibilité menées par ailleurs montrent que le modèle est relativement peu sensible à l'estimation d'autres paramètres, incluant la rugosité, le stockage en dépression, et la pente [Flanagan et al., 2017].

Outre les incertitudes importantes engendrées par l'estimation de $\mathrm{K}_{\mathrm{s}}$, les hypothèses simplificatrices du formalisme de Green et Ampt (1911) peuvent mener à un décalage de la simulation par rapport à la réalité. Comme mentionné précédemment, l'évapotranspiration depuis le sol n'est pas reproduite par le modèle ; le séchage du sol suite à une pluie est modélisé comme le retour de la teneur en eau à sa valeur initiale après une durée fixe, ce qui, de fait, ne permet pas la prise en compte de la variabilité de l'humidité du sol en fonction des saisons et des conditions météorologiques. Quoi qu'il en soit, l'effet du choix de la teneur en eau initiale est relativement faible : le fait de considérer un sol initialement sec (teneur en eau résiduelle), plutôt qu'un sol à la capacité au champ, augmente de 0,02 (resp. 0,04) le ratio entre volume infiltré et volume total arrivant sur la bande enherbée du segment 1 (resp. segment 2).

De plus, l'hypothèse d'une colonne de sol semi-infinie implique que la vitesse d'infiltration de l'eau ne sera jamais plus faible que la conductivité hydraulique à saturation tant que l'eau est présente en surface. En réalité, au niveau du deuxième segment, la structure de la chaussée présente un obstacle à l'infiltration à une profondeur d'au plus $30 \mathrm{~cm}$ sous la bande enherbée. En cas de périodes de pluie prolongées, il est possible que la couche de sol se sature entièrement, réduisant ainsi le volume total pouvant s'infiltrer ; le modèle, qui ne peut prendre en compte ces conditions, aura tendance à surestimer l'infiltration dans la bande enherbée pendant ces périodes. On ne rencontre pas le même problème sur le segment 1 qui est drainé.

Une limitation finale du modèle réside dans la représentation idéalisée du fonctionnement de la bande enherbée. On fait l'hypothèse (i) d'une répartition parfaite de l'eau sur chaque bassin versant, et (ii) d'une homogénéité des propriétés du sol. Ce faisant, on ne prend pas en compte la présence possible de chemins préférentiels de l'eau, aussi bien en surface qu'à l'intérieur du sol. Si le fonctionnement réel du système diffère de ce fonctionnement idéal, les résultats de modélisation perdront en représentativité. 


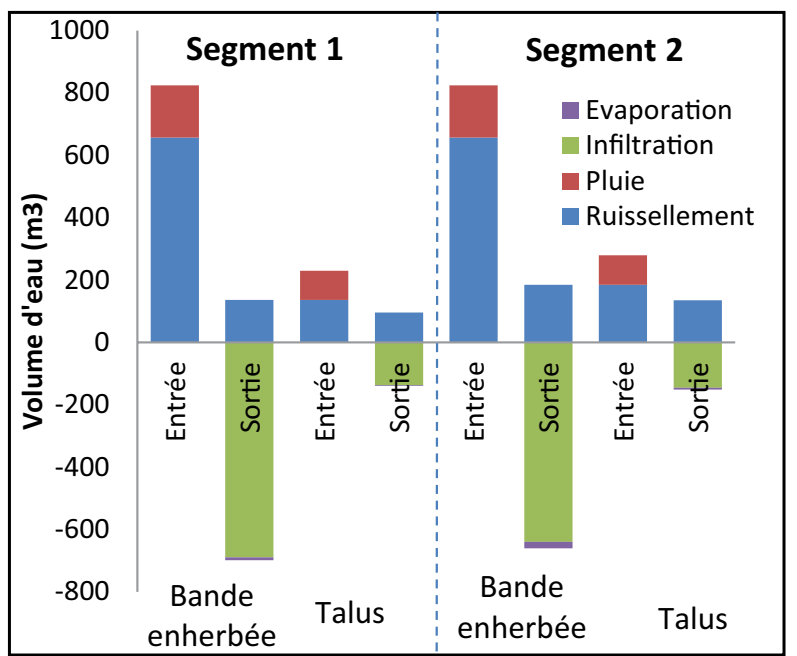

Figure 3 : Bilan hydrologique sur la bande enherbée et le talus des deux segments

En l'absence de données de validation, les sources d'incertitudes et les limitations du modèle obligent à une certaine prudence dans l'interprétation des résultats de modélisation. Dans le cas du site d'étude de Compans, où les observations sur site laissent à penser que le ruissellement atteint rarement le fossé filtrant, les résultats des simulations permettent de conclure que ce fonctionnement serait normal pour une bande enherbée possédant cette géométrie et ce type de sol, dans des conditions atmosphériques typiques du climat d'Ile-de-France ; en revanche, il n'est guère possible de conclure à l'exactitude du bilan hydrologique simulé.

Malgré les limitations inhérentes à ce type d'approche par modélisation conceptuelle, un outil tel que SWMM présente une certaine utilité pour mieux appréhender le fonctionnement hydrologique de ce type de dispositif, et ainsi optimiser la conception de nouveaux ouvrages ou systèmes d'ouvrages en série. En fournissant un ordre de grandeur de la sollicitation relative des différentes parties du système, le modèle permet de prévoir un dispositif de traitement à

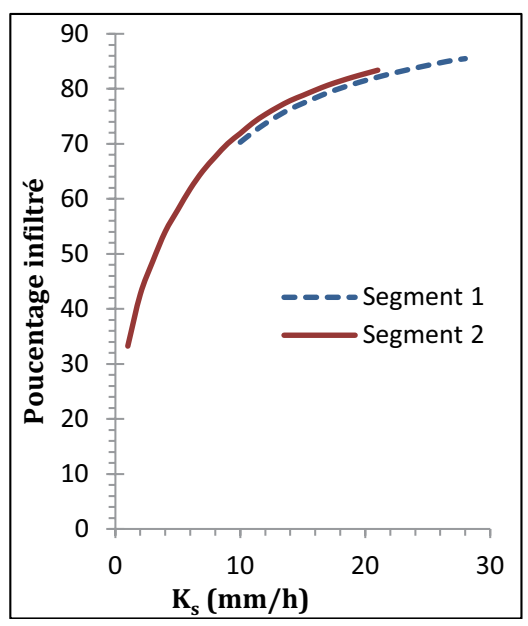

Figure 4 : Pourcentage de l'eau de ruissellement infiltrée dans la bande enherbée en fonction de sa conductivité hydraulique à saturation

l'emplacement où il sera le plus utile, c'est-à-dire là où il pourra intercepter la plus grande partie des flux d'eau et de polluants. Les présents résultats suggèrent en particulier que cet emplacement ne correspond pas à l'endroit où l'on prévoit le stockage d'eau en cas de pluie exceptionnelle, mais se situe plus proche de l'entrée de l'ouvrage. Pour des ouvrages existants, un tel modèle permet d'acquérir une compréhension du fonctionnement " normal » de l'ouvrage, que l'on peut ensuite comparer aux observations de terrain ou aux analyses de sol pour identifier des comportements inattendus.

Variabilité de la contamination en surface. Les tendances observées sont relativement similaires pour les trois métaux étudiés, et ce, malgré des spéciations potentiellement différentes dans le ruissellement - à titre d'exemple, le plomb est connu pour être majoritairement sous forme particulaire, tandis que la fraction dissoute est généralement plus importante pour le zinc [Gromaire-Mertz et al., 1999]. Leur distribution dans le sol superficiel de la bande enherbée (Figures $5 \mathrm{a}$ et $5 \mathrm{~b}$ ) atteste d'une accumulation significative

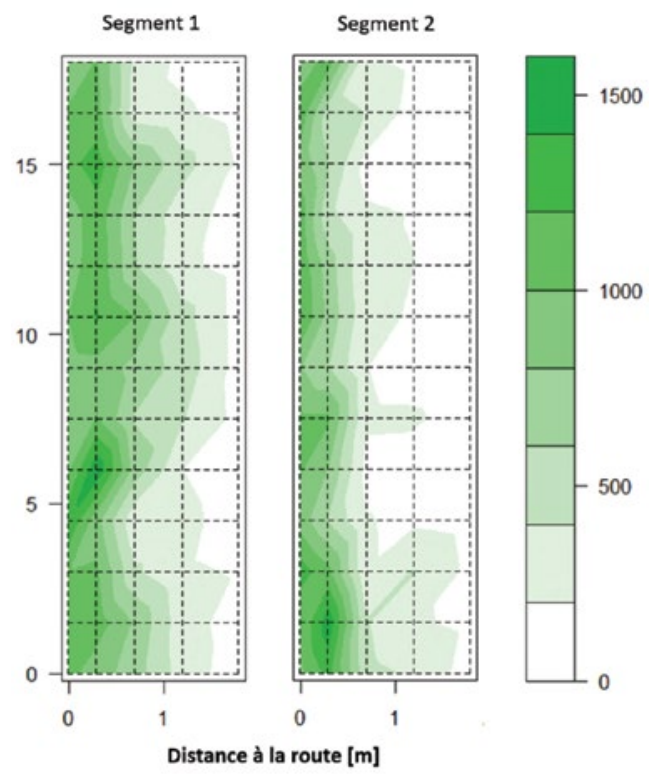

(a)

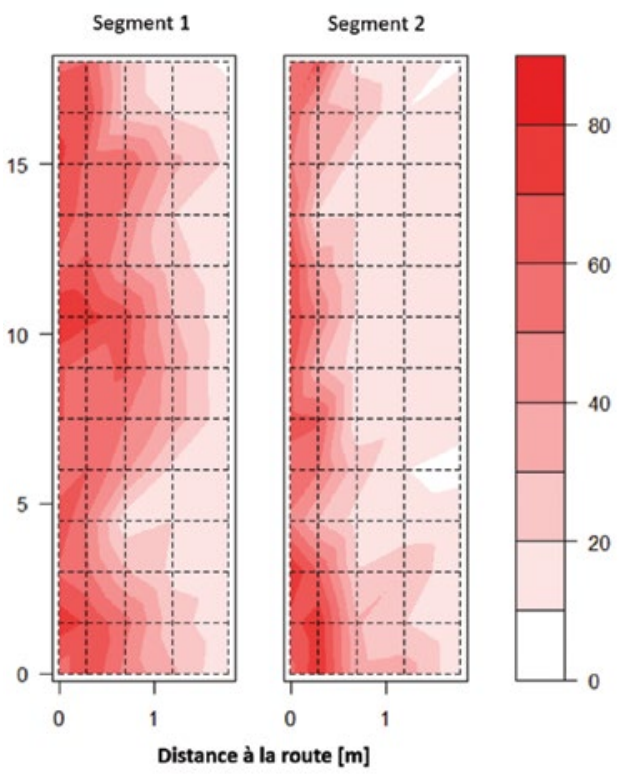

(b)

Figure 5 : Cartographies des concentrations en (a) zinc et (b) plomb [mg/kg] mesurées sur chacun des deux segments. La route se situe dans la zone $x<0$. 
de contaminants dans les 30 centimètres les plus proches de la route, c'est-à-dire de la zone d'arrivée de l'eau (située à l'abscisse $\mathrm{x}=0$ ), suivie d'une diminution des teneurs à mesure que l'on s'en éloigne. À cette décroissance caractéristique, s'ajoute une variabilité latérale des concentrations - qui fluctuent d'un facteur 1,5 à 4 entre des points équidistants de la chaussée. La contamination apparaît plus localisée sur le segment 2 , où les teneurs décroissent plus rapidement avec la distance à la route, que sur le segment 1 (Figure 6).

Les teneurs mesurées sur le segment témoin, où une bordure en béton empêche l'arrivée du ruissellement de voirie, permettent d'évaluer le bruit de fond géochimique du site, auquel s'ajoute la contribution des autres sources de contamination que sont les projections d'eau depuis la chaussée, la présence de la glissière de sécurité, et les retombées atmosphériques ; ces teneurs seront considérées ici comme des valeurs de " référence ". Les concentrations en cuivre $(23-41 \mathrm{mg} / \mathrm{kg})$ et plomb $(9-24 \mathrm{mg} / \mathrm{kg})$ ne montrent pas de tendance statistiquement significative avec la distance à la route (ANOVA à un facteur, $p>0,05$ ), contrairement au zinc $(67-237 \mathrm{mg} / \mathrm{kg})$ dont les concentrations sont plus élevées à proximité de la route, ce qui est probablement dû à la fraction des précipitations qui ruisselle sur la glissière galvanisée (Figure 6). En moyenne, le cuivre et le zinc sont détectés dans des concentrations supérieures aux valeurs médianes dans les sols de la petite région agricole correspondant à Compans $(15,5$ et $56,16 \mathrm{mg} / \mathrm{kg}$, respectivement), tandis que le plomb en est assez proche $(19,3 \mathrm{mg} / \mathrm{kg}$ ) (données issues de la Base de Données Eléments Traces Métalliques - BDETM).

Quoi qu'il en soit, les valeurs mesurées sur le segment témoin représentent de facto moins de $15 \%$ des concentrations maximales sur les segments 1 et 2 , ce qui prouve une influence significative des apports de polluants par le ruissellement de surface. La comparaison des niveaux de contamination observés sur ces trois segments permet d'évaluer la contribution « nette » de l'infiltration et de la sédimentation des métaux particulaires à la pollution globale du site (Figure 6). Au seuil $p=1 \%$, les concentrations en $\mathrm{Cu}$ et $\mathrm{Zn}$ sont significativement différentes entre le segment témoin et les deux autres segments, jusqu'à $1,2 \mathrm{~m}$ de la route ; pour $\mathrm{Pb}$ en revanche, la différence devient non significative à $1,2 \mathrm{~m}$ (segment 1 ) voire $70 \mathrm{~cm}$ (segment 2) de la route, ce qui pourrait être dû aux concentrations plus faibles de $\mathrm{Pb}$ dans le ruissellement par rapport à $\mathrm{Cu}$ et $\mathrm{Zn}$, ou à sa nature plus particulaire. En d'autres termes, la contamination due au ruissellement de voirie n'est visible que sur une partie de la bande enherbée, à l'autre extrémité de laquelle on retrouve des valeurs comparables aux teneurs de référence.

Ces résultats suggèrent ainsi que la quasi-totalité du flux de cuivre, plomb, et zinc, est retenue sur la bande enherbée, et n'atteint pas le fossé filtrant. Comme cela a été mentionné à la section précédente, la pluviométrie annuelle de la région parisienne est dominée par des événements pluvieux de faible intensité - responsables de la majorité du flux polluant - lors desquels les volumes générés peuvent s'infiltrer " localement » à proximité de la zone d'arrivée de l'eau, sans nécessairement recouvrir la surface totale de la bande enherbée. En d'autres termes, à l'échelle annuelle, la sollicitation hydrologique d'un tel ouvrage est variable dans l'espace. Dans le cas idéal d'un sol aux propriétés parfaitement homogènes sur toute la bande enherbée, cette sollicitation tend à décroître avec la distance à la route. Dans un cas réel, la structure de la contamination du sol de surface porte la signature conjointe des flux d'infiltration non-uniformes et de la sédimentation des métaux particulaires, mais la variabilité latérale des teneurs nous fournit des indications supplémentaires sur les hétérogénéités de l'écoulement sur la bande enherbée.

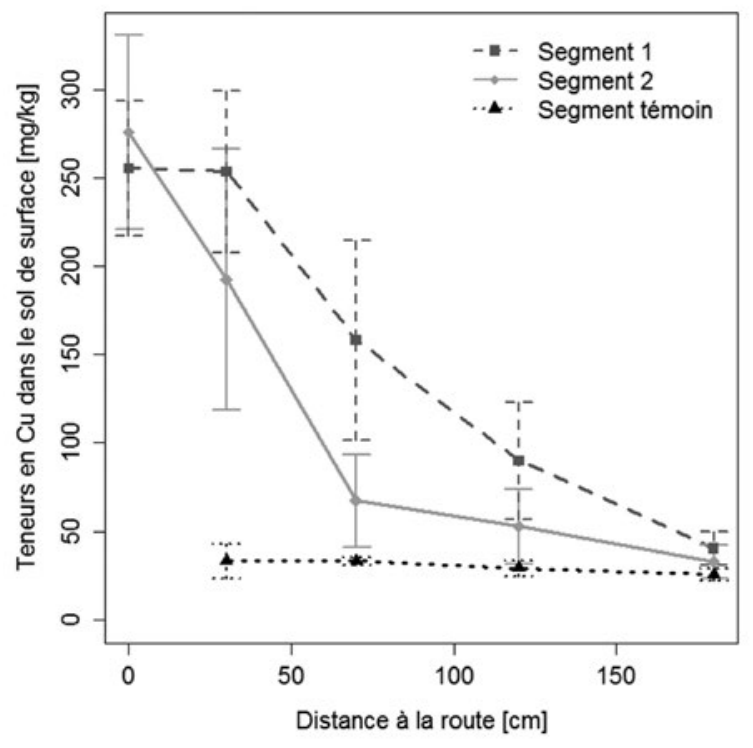

(a)

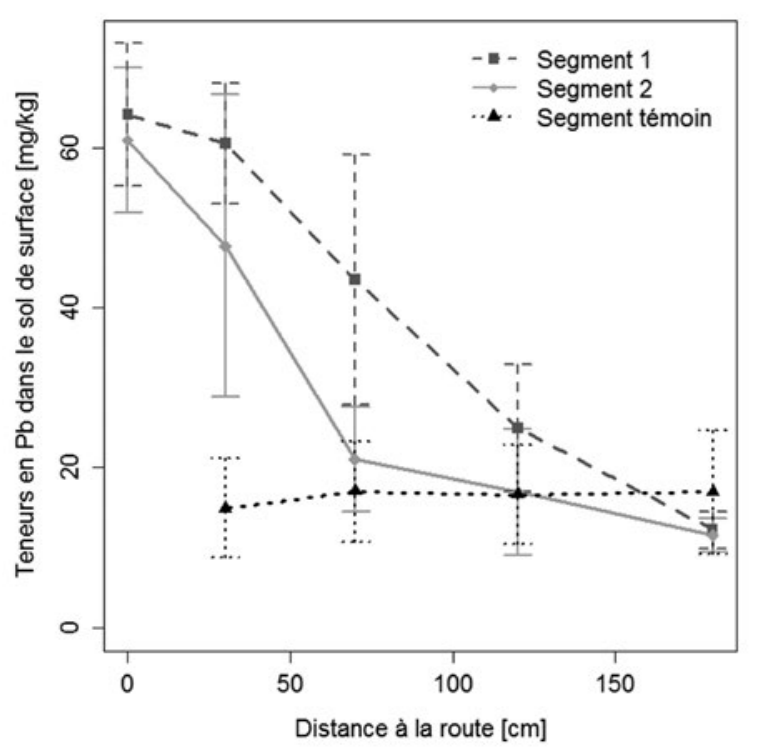

(b)

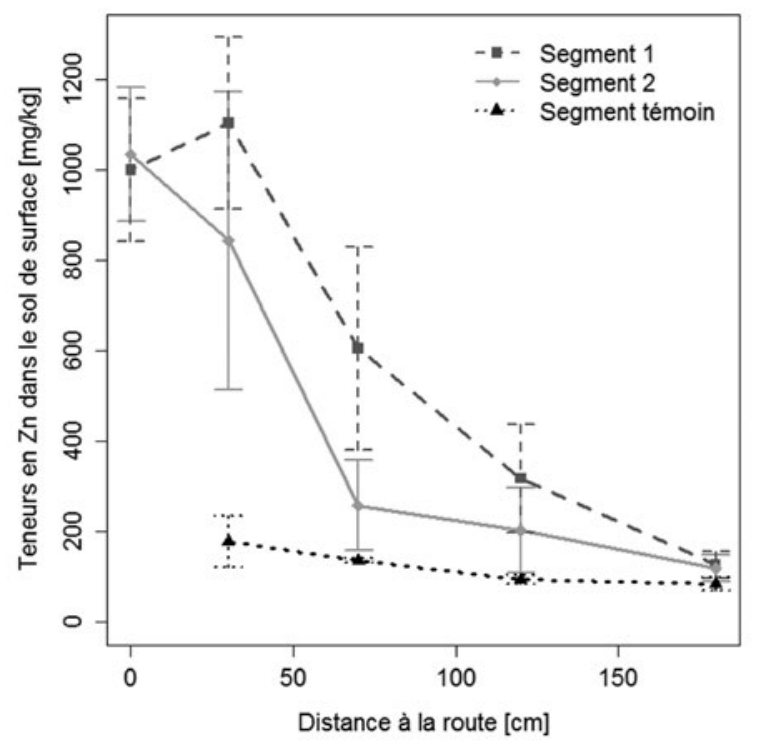

(c)

Figure 6 : Concentrations moyennes ( \pm écart-type) en (a) cuivre, (b) plomb, et (c) zinc [mg/kg], représentées en fonction de l'éloignement à la route sur chacun des segments échantillonnés. 
On a déjà mentionné le fait que la contamination était plus localisée sur le segment 2, dont le sol est limono-argileux, que sur le segment 1 , de texture sablo-limoneuse. Cette observation peut sembler contradictoire avec l'interprétation précédente, dans la mesure où une conductivité hydraulique plus élevée devrait favoriser une infiltration plus " locale » du ruissellement. De fait, des inspections visuelles du site par temps de pluie montrent très clairement (i) un dénivelé plus marqué entre la couche de roulement de la route et le sol de la bande enherbée sur le segment 1 que sur le segment 2, (ii) une accumulation de dépôt en bordure de bande enherbée bien plus importante sur le segment 2 que le segment 1 , formant un monticule linéaire de $2-3 \mathrm{~cm}$ de hauteur, et (iii) la formation d'un écoulement longitudinal, en particulier lors de phases de pluie intense, plus marqué sur le segment 2 que le 1 . Ainsi, il est probable qu'un flux de métaux différent soit arrivé sur les deux segments au cours des 4 années de fonctionnement de l'ouvrage. Par ailleurs, il se pourrait que la stagnation de l'eau à l'entrée de la bande enherbée du segment 2 favorise la formation d'un écoulement préférentiel à l'interface entre la structure de la route et le sol de la bande enherbée.

La cartographie à haute résolution spatiale $\left(1,8\right.$ point $\left./ \mathrm{m}^{2}\right)$ de la contamination du sol par différents polluants ubiquistes en milieu routier, apparaît donc comme un moyen original d'appréhender le fonctionnement des ouvrages d'infiltration, approche complémentaire à la simulation hydrologique, dans le sens où elle permet de dépasser les hypothèses simplificatrices inhérentes au modèle, pour accéder à une vision fine des hétérogénéités de l'infiltration et/ou du dépôt de particules. Toutefois cette approche ne peut être appliquée qu'après une durée de fonctionnement suffisante pour permettre un cumul notable de contaminants dans le sol. De plus, la contamination du sol donne une information sur les cheminements les plus fréquents de l'eau sans une résolution temporelle. Il est à noter que les métaux ne sont pas des traceurs parfaits de l'infiltration de l'eau. La fraction particulaire (majoritaire) peut être retenue par filtration mécanique au cours de l'infiltration ou par déposition pendant que l'eau ruisselle en surface, alors que le devenir de la fraction dissoute est gouverné par des processus d'adsorption à la matrice solide, dont les propriétés de rétention dépendent des caractéristiques du sol.

Variabilité de la contamination en profondeur. L'échantillonnage du sol par carottage a permis de compléter la compréhension de la distribution de la contamination métallique à la surface avec une vision de sa répartition en profondeur le long de quatre transects. Une fois encore, les trois métaux présentent des tendances très similaires, représentées dans le cas du cuivre sur la Figure 7. Rappelons que ces traitements cartographiques reposent sur l'hypothèse fondamentale d'une décroissance linéaire des concentrations avec $z$ au sein de chaque échantillon, ce qui a permis de faire correspondre concentrations et profondeurs moyennes, puis d'interpoler ces données moyennées. Dans le cas spécifique d'ouvrages conçus pour l'infiltration du ruissellement urbain ou routier, il existe quelques investigations verticales dont la résolution est suffisante pour valider cette hypothèse [Tedoldi et al., 2016]. Mikkelsen et al. [1996], qui ont prélevé des carottes de sol dans un accotement et les ont subdivisées en sous-échantillons de $2 \mathrm{~cm}$, ont effectivement mis en évidence une décroissance linéaire des concentrations en métaux au sein d'un même horizon de sol ; toutefois, les auteurs ont observé des ruptures abruptes à l'interface entre deux horizons - cas qui ne concerne pas la présente étude car les carottes ont été prélevées dans un matériau homogène. De même, Jones et Davis [2013], qui ont collecté des échantillons tous les $2,5 \mathrm{~cm}$ dans une cellule de biofiltration, ont démontré une décroissance rapide et approximativement linéaire des concentrations sur les 7,5 premiers centimètres, en-dessous desquels on retrouve des valeurs uniformes et comparables aux concentrations initiales du matériau.

Sur le premier segment, on observe un profil où les concentrations en surface ainsi que l'étendue verticale de la contamination diminuent avec la distance à la route. La contamination au niveau du deuxième segment apparaît encore plus localisée, aussi bien à la surface qu'en profondeur. Les concentrations mesurées au niveau de la structure de la route sont toujours plus faibles que dans les échantillons sus-jacents, ce qui ne soutient pas l'hypothèse d'un
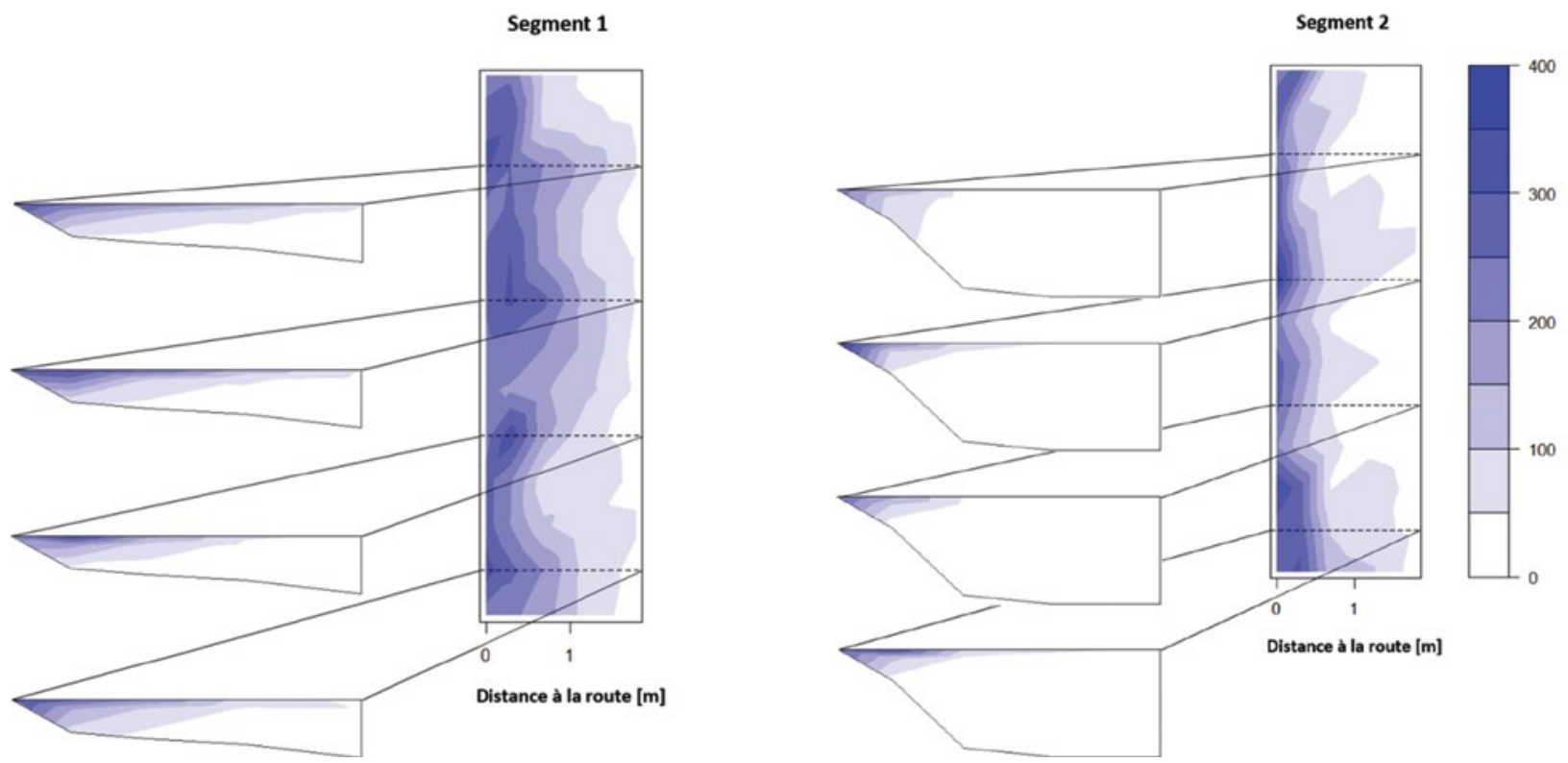

Figure 7 : Cartographie des concentrations en cuivre $[\mathrm{mg} / \mathrm{kg}]$ à la surface en en profondeur, mesurées sur chacun des deux segments. La route se situe dans la zone $x<0$. 
court-circuitage de la surface de la bande enherbée par un écoulement préférentiel le long de cette structure. En outre, la totalité du segment 2 demeure visiblement moins contaminée que le segment 1 , ce qui est cohérent avec les observations de terrain mentionnées dans la section précédente.

La contamination en métaux fournit une information $a$ posteriori sur la répartition des flux polluants, "intégrée " dans le temps depuis la mise en place du système, mais ne renseigne pas sur le comportement de la bande enherbée dans des conditions particulières. En d'autres termes, de telles observations ne sont pas incompatibles avec la présence occasionnelle d'écoulements préférentiels, engendrés notamment lors des phases de retrait du sol après une période sèche ; elles indiquent seulement que ce n'est pas le fonctionnement habituel du système. De la même façon, cette approche ne permet ni de reconstituer l'évolution du fonctionnement du système avec la formation du dépôt de sédiments, ni d'identifier la fréquence à laquelle l'eau parvient à dépasser la couche de sédiments dans l'état actuel.

\section{CONCLUSIONS}

Afin d'aborder le fonctionnement hydrologique d'un système de bande enherbée suivie d'un fossé filtrant pour la gestion à la source des eaux de ruissellement, deux méthodes ont été proposées et appliquées à un site réel : la simulation du fonctionnement hydrologique à long terme, à l'aide d'un modèle simplifié mis en œuvre sur le logiciel US EPA SWMM, et l'établissement d'une cartographie détaillée de la contamination du sol en métaux à l'aide de la spectrométrie de fluorescence $\mathrm{X}$, une méthode permettant de réaliser des analyses semi-quantitatives des teneurs en métaux à faible coût.

Pour le site d'étude, composé d'une bande enherbée suivie d'un fossé filtrant, les résultats obtenus via ces deux méthodes corroborent l'hypothèse élaborée à partir d'observations de terrain : pour des événements pluvieux courants, auxquels est associée la plus grande partie du flux polluant annuel, l'eau de ruissellement est gérée majoritairement par la partie de l'ouvrage la plus proche de la route : la bande enherbée. Même au sein de celle-ci, le flux polluant se concentre à proximité de la route. L'étude de la distribution de la contamination permet également de mettre en avant un dysfonctionnement au niveau de l'un des segments étudiés, dû à un dépôt de sédiments routiers, ainsi qu'à la formation de matière organique en surface, tous deux formant un obstacle à l'écoulement sur la bande enherbée. Ces phénomènes sont inhérents au fonctionnement d'une bande enherbée en contexte routier. Par conséquent, il est difficile de maintenir dans le temps les conditions idéales d'alimentation homogène de l'ouvrage.

L'approche de modélisation est utile, en premier lieu, comme une méthode a priori, permettant d'anticiper le bilan hydrologique « normal » du système pour une géométrie, un type de sol et des conditions climatiques donnés, et ainsi d'optimiser sa conception. En outre, le modèle pourrait fournir des indications sur la variabilité du fonctionnement de l'ouvrage pour différents types d'événements pluvieux. Il est néanmoins limité par l'incertitude importante engendrée par l'estimation de la conductivité hydraulique. Il implique également des hypothèses fortes sur le fonctionnement idéal du système ; le modèle aura un pouvoir prédictif très limité si ces hypothèses ne sont pas valables (par exemple en présence d'écoulements préférentiels ou d'obstacles à l'écoulement superficiel). Ce modèle représente uniquement les flux d'eau dans le système. Il n'est pas évident qu'on puisse tirer des relations directes entre flux d'infiltration et répartition de la pollution, dans la mesure où les polluants particulaires peuvent être retenus par décantation avant que toute l'eau ne se soit infiltrée. Quoi qu'il en soit, ce constat va dans le sens d'une rétention encore plus importante des polluants sur la bande enherbée que ce que prévoit la distribution des flux d'infiltration.

La méthode de l'échantillonnage du sol permet d'acquérir une vision de l'arrivée réelle du flux de contaminants sur le système. Le principal apport de cette méthode est sa capacité à révéler la variabilité de la contamination au sein de la bande enherbée, y compris en cas de fonctionnements inattendus (hétérogénéités, obstacles à l'écoulement) avec une fine résolution spatiale. On peut ainsi l'utiliser pour évaluer la pertinence des hypothèses prises dans le modèle hydrologique, et pour comparer la distribution réelle de la contamination à ce qu'on attendrait du modèle. Elle est limitée par son caractère $a$ posteriori - on ne peut l'utiliser qu'après une période suffisamment longue pour que la quantité de métaux accumulée dans le sol soit significativement différente du bruit de fond géochimique local. De plus, elle donne une information globale sur le fonctionnement de l'ouvrage depuis sa mise en place (i.e. sans résolution temporelle), ne permettant pas de mettre en avant des comportements événementiels ni des évolutions possibles du fonctionnement $d u$ système - à moins de mener plusieurs campagnes d'échantillonnage espacées dans le temps. Enfin, ce type de méthode ne permet pas de faire la distinction entre les contributions de l'infiltration et de la décantation dans la rétention des polluants particulaires, ce qui est le cas de la plupart des polluants présents dans le ruissellement de voirie.

Les deux méthodes présentées fournissent des informations permettant de mieux aborder le fonctionnement d'un ouvrage de gestion à la source des eaux de ruissellement de voirie, chacune avec ses propres limitations. Les apports possibles de chaque méthode étant différents, elles peuvent être appliquées conjointement et de manière complémentaire.

\section{REMERCIEMENTS}

Cette étude a été conduite dans le cadre de l'Observatoire de Polluants Urbains de la région Parisienne (OPUR) et le projet de recherche ROULEPUR. Les auteurs remercient vivement les partenaires d'OPUR (AESN, SIAAP, CD92, CD93, CD94, Ville de Paris), l'ONEMA et l'Agence de l'Eau Seine-Normandie pour leur soutien financier. Nous sommes également reconnaissants au Conseil Départemental de Seine-et-Marne, en particulier Éric Thomas et Tina Ratovelomanana, pour leur collaboration et leur soutien technique.

\section{BIBLIOGRAPHIE}

Bastien N., Arthur S., Wallis S., Scholz M. (2010) - The best management of SuDS treatment trains: a holistic approach. Water Science \& Technology, 61(1), 263-272.

Davis A.P., Hunt W.F., Traver R.G., Clar M. (2009) - Bioretention technology: Overview of current practice and future needs. Journal of Environmental Engineering, 135(3), 109-117.

Flanagan K., Branchu P., Ramier D., Gromaire M.-C. (2017) Evaluation of the relative roles of a vegetative filter strip and a biofiltration swale in a treatment train for road runoff. Water Science and Technology, 75(4), 987-997. 
Fletcher T.D., Shuster W., Hunt W.F., Ashley R., Butler D. Arthur S., Trowsdale S., Barraud S., Semadeni-Davies A., Bertrand-Krajewski J.-L., Mikkelsen P.S., Rivard G., Uhl M., Dagenais D., Viklander M. (2014) - SUDS, LID, BMPs, WSUD and more - The evolution and application of terminology surrounding urban drainage. Urban Water Journal, 12(7), 525-542.

GironÁs J., Roesner L., Davis J. (2009) - Storm Water Management Model Applications Manual. United States Environmental Protection Agency, Cincinnati, Ohio, Etats-Unis.

Green H.W., Ampt G.A. (1911) - Studies on Soil Phyics. Part I.- The flow of air and water through soils. The Journal of Agricultural Science, 4, 1-24.

Gromaire-Mertz M., Garnaud S., Gonzalez A., Chebbo G. (1999) - Characterisation of urban runoff pollution in Paris. Water Science and Technology, 39(2), 1-8.

JoNES P.S., DAvIS A.P. (2013) - Spatial Accumulation and Strength of Affiliation of Heavy Metals in Bioretention Media. Journal of Environmental Engineering, 139, 479-487.

KANSO T. (2015) - Caractérisation hydrodynamique d'un accotement de voirie végétalisé (Master). Université Libanaise.
Lefevre G., Paus K.H., Natarajan P., Gulliver J.S., Novak P.J., Hozalski R.M. (2014) - Review of Dissolved Pollutants in Urban Storm Water and Their Removal and Fate in Bioretention Cells. Journal of Environmental Engineering, 141.

Mikkelsen P.S., Häfliger M., OChs M., TJell J.C., Jacobsen P., Boller M. (1996) - Experimental assessment of soil and groundwater contamination from two old infiltration systems for road run-off in Switzerland. Science of The Total Environment, 189-190, 341-347.

Petrucci G. (2012) - La diffusion du contrôle à la source des eaux pluviales urbaines. Confrontation des pratiques à la rationalité hydrologique. Thèse de doctorat en hydrologie urbaine. Paris : Université Paris Est, 391p.

RAMIER D. (2005) - Bilan hydrique des voiries urbaines : observations et modélisation.Nantes : Université de Nantes.

Rossman L. (2015) - Storm Water Management Model Reference Manual: Hydrology. United States Environmental Protection Agency, Cincinnati, Ohio, Etats-Unis.

Tedoldi D., Chebbo G., Pierlot D., Kovacs Y., Gromaire M.-C. (2016) - Impact of runoff infiltration on contaminant accumulation and transport in the soil/filter media of Sustainable Urban Drainage Systems: A literature review. Science of The Total Environment, 569-570, 904-926. 